\title{
SIRT4 Overexpression Promotes the Inhibition of Cell Proliferation and Invasion in Prostate Cancer
}

\author{
*Abdul-Nazif Mahmud ${ }^{1}$ Feilun Cui ${ }^{1}$, Xue Boํㄹ Jianpeng $\mathrm{Hu}^{1}$, Tingjun Liu², Muslimat Kehinde Adebis ${ }^{3}$, Said \\ Abdulrahman Salim ${ }^{4}$, Aaron Gia Kanton ${ }^{4}$
}

\begin{abstract}
${ }^{* 1}$ Department of Urology, Affiliated People’s Hospital of Jiangsu University, Zhenjiang, Jiangsu, People’s Republic of China drsweetmom@mail.com,pdcuifeilun@163.com ${ }^{2}$ School of Medicine, Jiangsu University, Zhenjiang, Jiangsu, People’s Republic of China 2258246591@qq.com

${ }^{3}$ Department of Neurology, Affiliated Hospital of Jiangsu University, Zhenjiang, Jiangsu, People's Republic of China kennyades21@icloud.com

${ }^{4}$ Department of General Surgery, Affiliated Hospital of Jiangsu University, Zhenjiang, Jiangsu, People’s Republic of China Said_salim@live.com
\end{abstract}

\begin{abstract}
Background: SIRT4 is among the few characterized sirtuin groups of nicotinamide adenine dinucleotidedependent enzymes base in the mitochondria which facilitates several significant processes at the cellular level including stress response, metabolism, and longevity. SIRT4 can suppress and inhibits the growth, proliferation, and transformation of tumor cells such as colorectal and gastric tumors by suppressing glutamine anaplerosis, but no research reveals the roles and functions SIRT4 plays in the development of a prostate tumor.

Methods: Overexpression of SIRT4 in prostate cancer cell lines LNCaP was determined using RT-qPCR and western blot analyses. CCK-8 and transwell analysis were utilized to establish SIRT4 overexpression effects on cell proliferation and cell invasion respectively.

Results: This study first established SIRT4 overexpression in prostate cancer cell lines LNCaP, functional experiment such as CCK-8 assay and transwell assay revealed that overexpression of SIRT4 inhibits cell proliferation and invasion
\end{abstract}

Conclusions: SIRT4 has a tumor-suppressive function and may serve as a novel therapeutic target in prostate cancer

Keywords: Cell Invasion, LNCaP cells, Prostate cancer, SIRT4 and Tumour suppressor

\section{INTRODUCTION}

Prostate carcinoma is among the frequently diagnosed cancers globally[1] and also, the second main cause of death of cancer origin among men in the United States [2]. Compared to white men, African men are more prone and likely to be diagnosed at the advance stage of prostate cancer and also, have a higher mortality rate [ $\underline{3}]$. The incidence of prostate cancer increases with age. In 2018, the newly registered prostate cancer cases in the world accounted for approximately $7.1 \%$ of all new cancer cases among 
men. Prostate cancer, therefore, remains a major global health problem exploring the molecular mechanism of prostate cancer pathogenesis is still very essential to help develop new and effective treatment methods for prostate tumors. Sirtuin family consists of SIRT1-SIRT7[4]. Sirtuin serves a vital function in different cellular processes such as mitochondrial biosynthesis, fatty acid oxidation, insulin secretion, lipid metabolism, cellular stress response, aging, and apoptosis. It is revealed in a recent report that, the sirtuin group displays ADP-ribosylase and ADPdeacetylase activities [5-7]. Out of the 7 member family of sirtuin, (SIRT1, 6 and 7) have been established to be situated in the nucleus of the cell while (SIRT2 and SIRT3) in the cytoplasm and lastly, those in the cell mitochondrial are (SIRT4 and SIRT5)[ㅇ, $\underline{6}]$. Past genetic studies categorized the sirtuin family into four different classes. The SIRT4 is categorized to belong to class II of the sirtuin family [9]

Compare with other sirtuin families, SIRT4 expression has been found in numerous types of cells such as liver, testis, striated muscles, kidney vascular smooth muscle, and $\beta$ cells of the pancreas[10]. The activity of the SIRT4 sirtuin family group is located on ADP-ribosyltransferase instead of NAD+-dependent deacetylase activity[11]. A few research have reported that SIRT4 is involved in a vital role to regulate cellular metabolism and maintains genomic stability[12].

Other reports indicated that the inhibition of glutamine catabolism is regulated by SIRT4 following DNA damage. $[\underline{13}, \underline{14}]$. The flux of damage DNA is elevated through the pathway of pentose phosphate and declines the update of glutamine and the intermediates of TCA cycle levels. Glutaminase in the mitochondrial can catabolize glutamine to glutamate through the activities of mitochondrial GDH and AST [15]. A previous study showed that damage DNA induces the glutamine metabolism and anaplerosis by SIRT4 ADP-ribosylation and GDH inhibition[16].
Even though, the expression of SIRT4 in some cells and tissue is up-regulated following DNA damage, in several kinds of tumor cells the expression of SIRT4 was also found to be down regulated[17]. Reduction in expression of SIRT4 leads to elevation of glutaminedependent proliferation and stress-induced genetic instability, leading to a tumor with a phenotypic pattern. Lack of SIRT4 in a damaged DNA delayed in DNA repair as well as increase chromosomal aneuploidies demonstrating that SIRT4 could spontaneously preserve the damage of cells[18]. Other studies indicated that mice expressing no SIRT4 spontaneously develop lung cancer $[\underline{12}, \underline{19}]$.

In addition to the above, it has been established in current studies that, overexpression of SIRT4 in colorectal tumor cells caused an elevated expression of E-cadherin thereby impeding proliferation, migration, and invasion of colorectal tumor cells this is due to the function of SIRT4 as an inhibitor of glutamine catabolism. It was further established that, as the colorectal cancer invasion advances the expression level of SIRT4 becomes lower[무].

A recent report also indicated that glutamine supplementation promoted by the mammalian target of the rapamycin complex 1 (mTORC1) activates GDH, which requires SIRT4 transcriptional repression. Precisely, mTORC1 inhibits SIRT4 by destabilizing the connection of the cAMP-sensitive element 2 (CREB2). Besides, leucine is a key regulator of mTORC1 and SIRT4 can control its activity by reducing intracellular leucine levels [21]. Therefore, SIRT4 is an essential part of the DNA damage response pathway, which can regulate the metabolic obstruction of glutamine metabolism, cell cycle, and tumor suppression.

Furthermore, a clinical meta-analysis showed that the expression of SIRT4 mRNA was down-regulated in several malignant tumors and SIRT4 downregulation was closely correlated with numerous aggressive tumors such as gastric, breast, and 
colorectal cancers $[\underline{6}, \underline{20}, \underline{22}]$. The role of SIRT4 is, however, completely unknown in prostate tumors.

In this research, we established the expression of SIRT4 and assessed the significant roles of SIRT4 in suppressing LNCaP prostate cancer cell lines by impeding cell proliferation and invasion providing information about the possibility of considering SIRT4 as a new treatment target of prostate tumor.

\section{METHODS AND MATERIALS}

\section{Cell lines and culture}

LNCaP prostate tumor cell lines and normal prostate cell lines RPWE-1 were acquired from the cell bank of the Chinese Academy of Sciences (Shanghai, China). Cells were cultured in RPMI-1640 and KSM serum media (Gibco, Grand Island, NY, USA) comprising $100 \mathrm{U} / \mathrm{ml}$ penicillin, 10\% fetal bovine serum (FBS), $100 \mu \mathrm{g} / \mathrm{mL}$ streptomycin and $200 \mu \mathrm{M} \mathrm{L-}$ Glutamine (Gibco, USA), the cells were cultured at $37^{\circ} \mathrm{C}$ incubator with $5 \% \mathrm{CO}_{2}$.

\section{Plasmid and cell transfection}

Plasmids containing SIRT4 were acquired from the Biological Engineering Co., Ltd. (Shanghai, China). The expression of SIRT4 was first determined in LNCaP and RPWE-1 cells using standard molecular techniques with an empty vector as control. The following forward and reverse sequences of SIRT4 were used:

forward: 5'-AAGAGTTACAGCGCTTCATCACC-3' and reverse: 5'CGATTGCAATACTGAACATTGG-3'. Lipofectamine 3000 (Thermo Fisher Scientific, Inc., Waltham, MA, USA) was used in transfection. To generate stably transfected cells, the modified eagle medium (Opti-MEM) was changed to RPMI-1640 medium, which contained $100 \mu \mathrm{g} / \mathrm{ml}$ streptomycin, $10 \%$ fetal bovine serum, $100 \mathrm{U} / \mathrm{ml}$ penicillin, and $200 \mu \mathrm{M}$ of L-glutamine (Gibco, USA). The transfected cells were cultured in an incubator with $5 \% \mathrm{CO}_{2}$ at $37^{\circ} \mathrm{C}$. Following transfection for 48 hours, SRT4 expression was established by RT-qPCR and Western blot.

\section{RNA extraction and qRT-PCR}

TRIzol reagent (Invitrogen; Thermo Fisher Scientific, Inc.) was used to extract total RNA from the cells base on the instructions from the manufacturer. For RNA extraction. Prime Script RT Master Mix (Takara, Japan) was used to reverse transcribed $1 \mu \mathrm{g}$ cDNA base on the instructions from the manufacturer. Then, qRT-PCR was done using QuantStudio with SYBR green. Expression of Relative mRNA was normalized against GAPDH and the $2-\Delta \Delta \mathrm{Ct}$ method was used to analyze the relative expression level.

\section{Western blot analysis}

According to the manufacturer's instructions, total protein was extracted using RIPA buffer (Beyotime, Suzhou, China). Bradford test [23] was used to measure the concentration of protein. 10\% SDSPAGE gel electrophoresis was used to separate an equal quantity of protein samples which were then transferred to the nitrocellulose filter membrane (EMD Millipore, Billerica, MA, USA). 5\% skim milk mixed in $1 \times$ TBS containing $0.05 \%$ Tween-20 was used to block the membrane for 1 hour at room temperature. The following target antibodies PSA ABclonal (Wuhan, China), P53, GLUD-1 MTOR-1 (Abcam) and GAPDH (Sigma, USA) were used to incubate the membrane overnight at $4^{\circ} \mathrm{C}$. After that, it was washed 3 times with $1 \times$ TBST, and then placed in horseradish peroxidase-conjugated secondary antibody (Sigma, USA) at room temperature for 1 hour, and then washed with $1 \times$ TBST again for 10 minutes. An enhanced chemiluminescence system was used base on the instructions from the manufacturer to visualize the specific protein bands.

\section{CCK-8 assay}

To detect SIRT4 overexpression effect on cell proliferation, CCK-8 analysis was done base on the instructions from the manufacturer. Following transfection for $48 \mathrm{~h}$, cells were collected and seeded into 96 -well plates at a density of $4 \times 10^{3}$ well-1 and 
placed in an incubator at $37^{\circ} \mathrm{C}$ using $5 \% \mathrm{CO}_{2}$ for 0,24 , 48 , and $72 \mathrm{~h}$. Afterward, $20 \mu \mathrm{L}$ of CCK8 reagent Biological Engineering Co., Lt (Shanghai) was added to each well of the SIRT4 overexpression group and control group, and the plate was placed back in the incubator for additional 2hours. Multiplate reader (BioTek, Winooski, VT, USA) was utilized to measure the absorbances at $450 \mathrm{~nm}$. Finally, Excel and GraphPad 6.01 were used to analyze half-maximal inhibitory concentration (IC50) values and draw IC50 curves.

\section{Transwell assay}

To establish the effect of SIRT4 overexpression on cell invasion, Transwell analysis was carried out using a Transwell chamber (Corning, NY, USA) base on the instructions from the manufacturer. $15 \mu \mathrm{L}$ Matrigel (Corning) was used to coat the Transwell inserts. $1 \times 10^{6}$ cells were seeded into the upper chamber of the transwell after transfection which contained $200 \mu \mathrm{L}$ RPMI-1640 serum-free medium and $600 \mu \mathrm{L}$ of RPMI-1640 comprising 200 $\mu \mathrm{M}$ L-Glutamine, $10 \%$ FBS, $100 \mu \mathrm{g} / \mathrm{mL}$ streptomycin and $100 \mathrm{U} / \mathrm{ml}$ penicillin was added to the lower chamber of the transwell and then placed in an incubator for $24 \mathrm{~h}$ with $5 \% \mathrm{CO}_{2}$ at $37{ }^{\circ} \mathrm{C}$. All cells in the upper chamber were removed using cotton swabs. $4 \%(\mathrm{v} / \mathrm{v})$ PFA was used to fix the cells that crossed the filter and stained using $0.1 \%$ $(\mathrm{w} / \mathrm{v})$ crystal violet. The cells were incubated again at a temperature ranging from $20-25^{\circ} \mathrm{C}$ for 20 minutes. Finally, the cells were taken randomly from 6 fields counted and analyzed with an inverted microscope (Leica Microsystems).

\section{RESULTS AND DISCUSSION}

RT-qPCR of SIRT4 gene expression in prostate tumor cells and Normal prostate cells

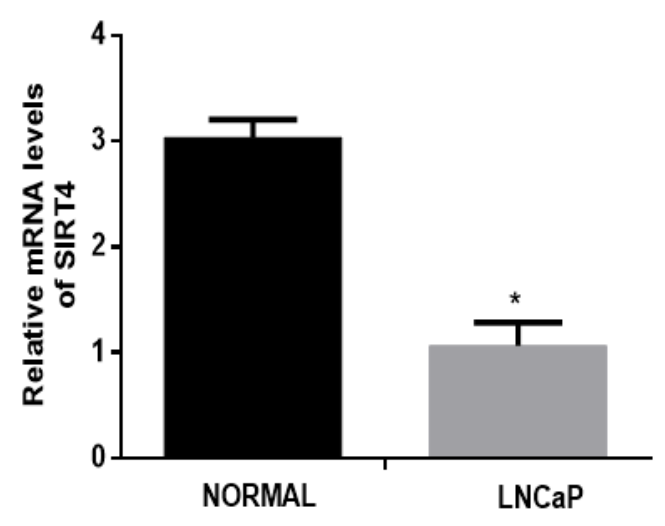

Fig. 1 The expression of SIRT4 gene was considerably lower in $L N C a P$ prostate cancer cells compared to the normal prostate cell (RWPE-1). . ${ }^{*} P<0.05$.

RT-qPCR of SIRT4 gene over-expression in LNCaP cells

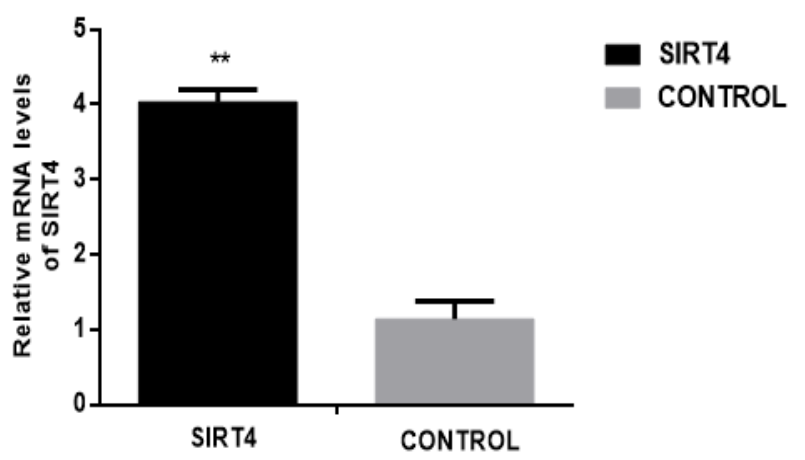

Figure 2 SIRT4 gene was transfected in the SIRT4 group of the $L N C a P$ cell lines and empty vector as a control group for $48 \mathrm{~h}$. The expression level in both groups was analyzed using $R T-q P C R$. SIRT4 gene significantly overexpressed in the SIRT4 group compare to the control. ${ }^{*}{ }^{*} P<0.01$

Growth of LNCaP cells after 48hours of transfection with overexpressed SIRT4 gene 


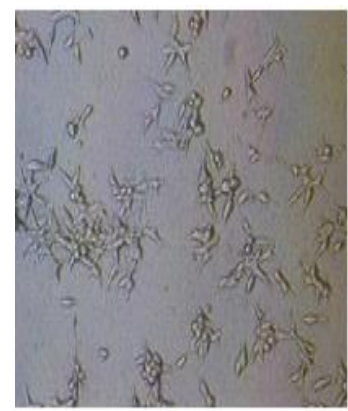

SIRT4

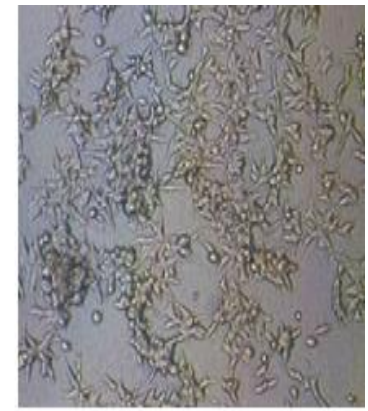

Control
Fig 3. The number of LNCaP cells in the SIRT4 overexpressed group considerably declined compared to the control group. Also, the cells in the overexpressed SIRT4 group looks more round and triangular compare to the control.

Effect of SIRT4 overexpression on cell proliferation.

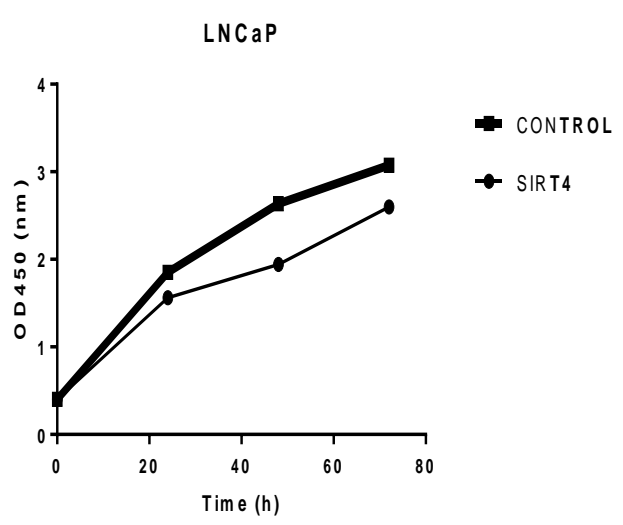

Figure 4 Cell proliferation was analyzed by the CCK- 8 assay. Cells overexpressing SIRT4 progressively decreased in growth compared to the control $P,<0.05$.

Effect of SIRT4 overexpression on cell invasion.
Transwell assay

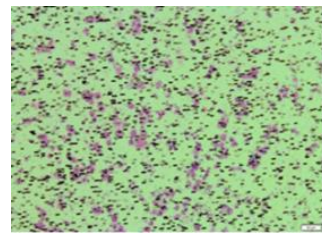

SIRT4

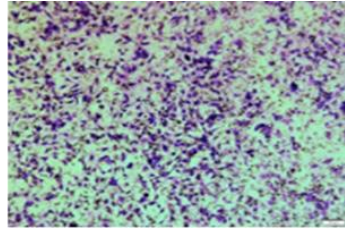

CONTROL

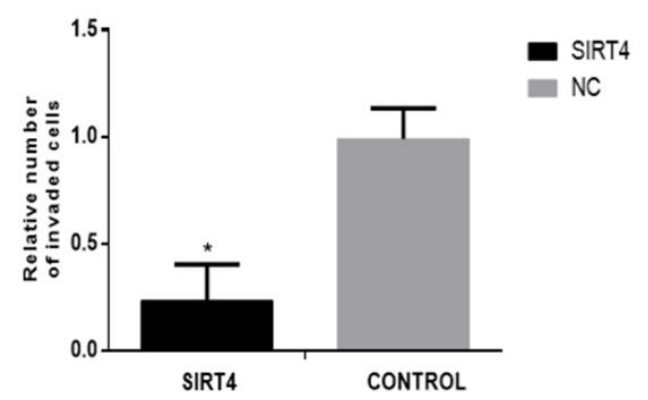

n cell proliferat

Fig. 5 Transwell analysis was utilized to determine the effect of SIRT4 on cell invasion. Stained cells that crossed the Matrigel were observed using a light microscope. The image was quantified and analyzed using GraphPad 6.0. The overexpressed SIRT4 group significantly inhibited the number of invaded cells compared with the control. ${ }^{*} p, 0.05$.

\section{RT-qPCR result of target genes}

The mRNA level of the target gene using qRT-PCR, genes such as MTOR and GLUD1 mRNA expression levels were detected

Fig. 6 RT-qPCR results of the target genes; Compared to the control group, the MTOR gene in the SIRT4 overexpression group was up-regulated ${ }^{*} \mathrm{p}<0.05$. However, compared to the control group, GLUD1 in the SIRT4 overexpression group was down-regulated. $\mathrm{p}>0.05$. 


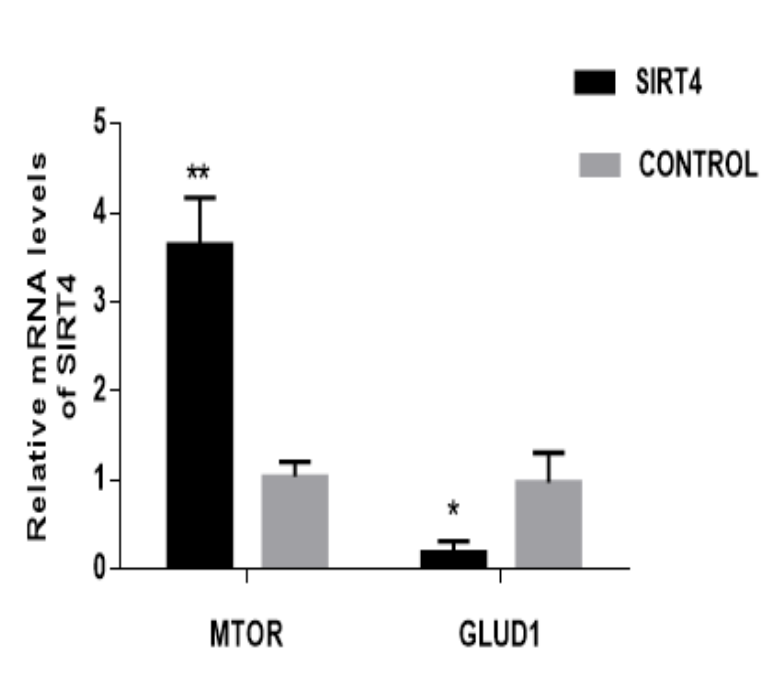

Fig. 6 The SIRT4 overexpressed group of mTOR was significantly increased in expression compared to the control " $" p<0.01$, However, the SIRT4 overexpressed group of GLUD1 was significantly decreased compared to the control " $p<0.05$.

\section{Western blot result of target proteins related to SIRT4}

The following targeted proteins MTOR and GLUD1 with GAPDH as housing keeping protein were detected. mTOR protein was significantly upregulated in the overexpressed SIRT4 group compare to the control group, However GLUD1 protein was downregulated in the overexpressed SIRT4 group compare to the control group indicating consistent expression at both the nucleic acid and protein levels.

\section{Western blot}

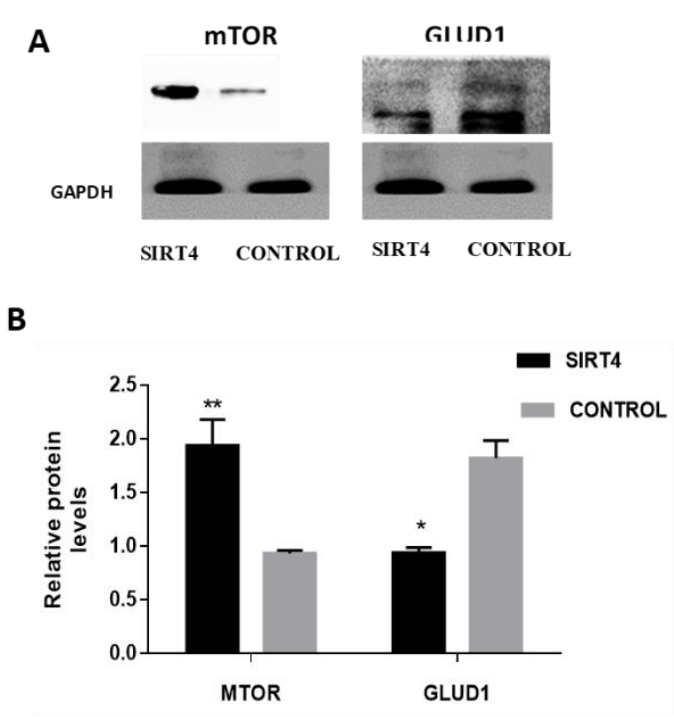

Fig. 7 Western blot result of target gene-related to SIRT was detected using western blot. The relative protein levels in control and cells overexpressing SIRT4 were analyzed. Data indicate mean \pm sd. of at least three independent experiments $m$ TOR $^{* *} p<0.01$, and GLUD1" $p<0.05$.

\section{DISCUSSION}

Past reports have discovered that SIRT4 is downregulated in numerous malignant growth cells including gastric and colorectal tumors $[\underline{24}, \underline{25}]$ yet the role of SIRT4 in prostate cancer cell lines has not been known. To explore the role of SIRT4 in prostate malignant cells, we first compared the SIRT4 expression in prostate malignant cell lines LNCaP and normal prostate cell lines RPWE-1 using RT-qPCR. The expression of the SIRT4 level was significantly lower in LNCaP cells compared to normal prostate cells $(\mathrm{P}<0.05)$. Transfection was then carried out to insert SIRT4 plasmid in one group and an empty vector as a control group in LNCaP cells. The expression of SRT4 was determined 48 hours after transfection using RT-qPCR. The expression level of cells transfected with the SIRT4 plasmid significantly 
increased in LNCaP cells compared to the control $(* *$ $\mathrm{P}<0.01)$.

After transfection, the condition of the cells was also observed under an inverted microscope to compare the growth changes of cells in both the SIRT4 overexpressed (SIRT4 OE) group and control. The number of LNCaP cells in the SIRT4 OE group was significantly reduced compared to the control group. The shape of cells in the SIRT4 OE group was also found to be more round and triangular indicating that SIRT4 overexpression plays a role in morphological changes and decreases the number of LNCaP cells.

A recent study showed that overexpression of SIRT4 in colorectal tumor cells inhibited cell proliferation $[\underline{24}, 25]$.To determine the function of overexpressed SIRT4 on prostate cancer cells, proliferation analyses were carried out in LNCaP cells. SIRT4 OE group significantly decreased the rate of cell growth compared to the control. This indicates that SIRT4 overexpression might play a role in impeding cell proliferation in prostate cancer cells.

A metastatic complication of a prostate tumor remains a significant challenge in the treatment of prostate malignant growth; the impact of metastatic phenotypes of SIRT4 in colorectal cancer was confirmed by substantial inhibition of cell invasion[4]]. To establish the effect of SIRT4 overexpression on cell invasion, Transwell analysis was carried out. The overexpressed SIRT4 group inhibited the number of invaded cells compared to the control group. We can confirm that overexpressed SIRT4 inhibits cell invasion in prostate cancer cell lines LNCaP.

Recent studies have shown that mammalian targets of rapamycin complex (mTORC1) promote glutamine supplementation to activate the transcriptional repression of SIRT4 required for GDH. To be precise, mTORC1 eliminates SIRT4 by breaking the association of cAMP-responsive element-binding 2 (CREB2). Furthermore, leucine is a key regulator of
mTORC1 and SIRT4 can control its effect by reducing intracellular leucine levels.[26]. Therefore, SIRT4 is an important part of the DNA damage response pathway, which can regulate metabolic obstruction of glutamine metabolism, cell cycle, and tumor suppression. In this study, the mRNA levels of mTOR and GLUD1 expression were determined by RT-qPCR and western blot. After transfection for $48 \mathrm{~h}$, the mRNA level of the overexpressed SIRT4 group and control was analyzed. The overexpressed SIRT4 group of mTOR was significantly increased in expression compared to the control ${ }^{* *} \mathrm{p}<0.01$. However, the SIRT4 overexpressed group of GLUD1 was significantly decreased compared to the control ${ }^{*} \mathrm{p}<0.05$.

To further assess the activity level of mTOR and GLUD1 at the protein level a western blot was conducted in control and cells overexpressing SIRT4. Data indicate $\operatorname{mTOR}^{* *} p<0.01$, and $\mathrm{GLUD} 1{ }^{*} p<0.0$. The expression levels of both genes at the protein and nucleic acid levels were consistent with previous studies indicating that both genes may play a similar role in prostate cancer.

\section{v. CONCLUSION}

This study merely investigated the regulation of SRT4 overexpression in prostate cancer cells, but the downstream regulation role of SIRT4 in a prostate cancer cell is not yet clear. The research findings indicate that SIRT4 can be stimulated to be overexpressed in prostate cancer cell lines LNCaP, SIRT4 overexpression acts as a tumor suppressor by inhibiting cell proliferation and invasion, the suppression of SIRT4 by target genes may be the underlying mechanism of inhabitation of proliferation and invasion in LNCaP cell lines. In conclusion, we suggest that SIRT4 may serve as a novel therapeutic target of prostate cancer. 


\section{FUNDING}

Social Development Plan of Jiangsu Province Standardization of key disease diagnosis and treatment project (BE2016715L)

\section{REFERENCES}

[1]. F. Bray, J. Ferlay, I. Soerjomataram, R. L. Siegel, L. A. Torre, and A. Jemal, "Global cancer statistics 2018: GLOBOCAN estimates of incidence and mortality worldwide for 36 cancers in 185 countries," CA: a cancer journal for clinicians, 2018.

[2]. R. L. Siegel, and K. D. Miller, "Cancer statistics, 2019," vol. 69, no. 1, pp. 7-34, Jan, 2019.

[3]. K. A. Cronin, A. J. Lake, S. Scott, R. L. Sherman, A. M. Noone, N. Howlader, S. J. Henley, R. N. Anderson, A. U. Firth, and J. Ma, "Annual Report to the Nation on the Status of Cancer, part I: National cancer statistics," Cancer, 2018.

[4]. H. Sun, D. Huang, G. Liu, F. Jian, J. Zhu, and L. Zhang, "sirT4 acts as a tumor suppressor in gastric cancer by inhibiting cell proliferation, migration, and invasion," OncoTargets and therapy, vol. 11, pp. 3959, 2018.

[5]. M. C. Haigis, R. Mostoslavsky, K. M. Haigis, K. Fahie, D. C. Christodoulou, A. J. Murphy, D. M. Valenzuela, G. D. Yancopoulos, M. Karow, and G. Blander, "SIRT4 inhibits glutamate dehydrogenase and opposes the effects of calorie restriction in pancreatic $\beta$ cells," Cell, vol. 126, no. 5, pp. 941-954, 2006.

[6]. G. Laurent, V. C. de Boer, L. W. Finley, M. Sweeney, H. Lu, T. T. Schug, Y. Cen, S. M. Jeong, X. Li, A. A. Sauve, and M. C. Haigis, "SIRT4 represses peroxisome proliferator-activated receptor alpha activity to suppress hepatic fat oxidation," Mol Cell Biol, vol. 33, no. 22, pp. 4552-61, Nov, 2013.
[7]. B. Liu, W. Che, J. Xue, C. Zheng, K. Tang, J. Zhang, J. Wen, and Y. Xu, "SIRT4 prevents hypoxia-induced apoptosis in $\mathrm{H} 9 \mathrm{c} 2$ cardiomyoblast cells," Cell Physiol Biochem, vol. 32, no. 3, pp. 655-62, 2013.

[8]. Y. Li, Y. Zhou, F. Wang, X. Chen, C. Wang, J. Wang, T. Liu, Y. Li, and B. He, "SIRT4 is the last puzzle of mitochondrial sirtuins," Bioorg Med Chem, vol. 26, no. 14, pp. 3861-3865, Aug 7, 2018.

[9]. N. Ahuja, B. Schwer, S. Carobbio, D. Waltregny, B. J. North, V. Castronovo, P. Maechler, and E. Verdin, "Regulation of insulin secretion by SIRT4, a mitochondrial ADP-ribosyltransferase," J Biol Chem, vol. 282, no. 46, pp. 33583-92, Nov 16, 2007.

[10]. E. Michishita, J. Y. Park, J. M. Burneskis, J. C. Barrett, and I. Horikawa, "Evolutionarily conserved and nonconserved cellular localizations and functions of human SIRT proteins," Mol Biol Cell, vol. 16, no. 10, pp. 4623-35, Oct, 2005.

[11].G. Huang, and G. Zhu, "Sirtuin-4 (SIRT4), a therapeutic target with oncogenic and tumorsuppressive activity in cancer," OncoTargets and therapy, vol. 11, pp. 3395, 2018.

[12]. S. M. Jeong, C. Xiao, L. W. Finley, T. Lahusen, A. L. Souza, K. Pierce, Y.-H. Li, X. Wang, G. Laurent, and N. J. German, "SIRT4 has tumorsuppressive activity and regulates the cellular metabolic response to DNA damage by inhibiting mitochondrial glutamine metabolism," Cancer cell, vol. 23, no. 4, pp. 450-463, 2013.

[13]. R. A. H. van de Ven, D. Santos, and M. C. Haigis, "Mitochondrial Sirtuins and Molecular Mechanisms of Aging," Trends Mol Med, vol. 23, no. 4, pp. 320-331, Apr, 2017.

[14]. L. Wang, H. Zhou, Y. Wang, G. Cui, and L. J. Di, "CtBP maintains cancer cell growth and metabolic homeostasis via regulating SIRT4," 
Cell Death Dis, vol. 6, no. 1, pp. e1620, Jan 29, 2015.

[15]. D. Komlos, K. D. Mann, Y. Zhuo, C. L. Ricupero, R. P. Hart, A. Y. Liu, and B. L. Firestein, "Glutamate dehydrogenase 1 and SIRT4 regulate glial development," Glia, vol. 61, no. 3, pp. 394408, Mar, 2013.

[16].K. A. Hershberger, A. S. Martin, and M. D. Hirschey, "Role of $\mathrm{NAD}(+)$ and mitochondrial sirtuins in cardiac and renal diseases," Nat Rev Nephrol, vol. 13, no. 4, pp. 213-225, Apr, 2017.

[17]. S. Shukla, A. Sharma, V. K. Pandey, S. Raisuddin, and P. Kakkar, "Concurrent acetylation of FoxO1/3a and p53 due to sirtuins inhibition elicit Bim/PUMA mediated mitochondrial dysfunction and apoptosis in berberine-treated HepG2 cells," Toxicol Appl Pharmacol, vol. 291, pp. 70-83, Jan 15, 2016.

[18].P. J. Fernandez-Marcos, and M. Serrano, "Sirt4: the glutamine gatekeeper," Cancer Cell, vol. 23, no. 4, pp. 427-8, Apr 15, 2013.

[19]. D. Germain, "Sirtuins and the Estrogen Receptor as Regulators of the Mammalian Mitochondrial UPR in Cancer and Aging," Adv Cancer Res, vol. 130, pp. 211-56, 2016.

[20]. M. Miyo, H. Yamamoto, M. Konno, H. Colvin, N. Nishida, J. Koseki, K. Kawamoto, H. Ogawa, A. Hamabe, and M. Uemura, "Tumoursuppressive function of SIRT4 in human colorectal cancer," British journal of cancer, vol. 113, no. 3, pp. 492, 2015.

[21]. A. Csibi, S. M. Fendt, C. Li, G. Poulogiannis, A. Y. Choo, D. J. Chapski, S. M. Jeong, J. M. Dempsey, A. Parkhitko, T. Morrison, E. P. Henske, M. C. Haigis, L. C. Cantley, G. Stephanopoulos, J. Yu, and J. Blenis, "The mTORC1 pathway stimulates glutamine metabolism and cell proliferation by repressing SIRT4," Cell, vol. 153, no. 4, pp. 840-54, May 9, 2013.
[22]. G. Huang, F. Cui, F. Yu, H. Lu, M. Zhang, H. Tang, and Z. Peng, "Sirtuin-4 (SIRT4) is downregulated and associated with some clinicopathological features in gastric adenocarcinoma," Biomedicine \& Pharmacotherapy, vol. 72, pp. 135-139, 2015.

[23].M. M. Bradford, "A rapid and sensitive method for the quantitation of microgram quantities of protein utilizing the principle of protein-dye binding," Analytical biochemistry, vol. 72, no. 12, pp. 248-254, 1976.

[24]. M. Miyo, H. Yamamoto, M. Konno, H. Colvin, N. Nishida, J. Koseki, K. Kawamoto, H. Ogawa, A. Hamabe, M. Uemura, J. Nishimura, T. Hata, I. Takemasa, T. Mizushima, Y. Doki, M. Mori, and H. Ishii, "Tumour-suppressive function of SIRT4 in human colorectal cancer," Br J Cancer, vol. 113, no. 3, pp. 492-9, Jul 28, 2015.

[25]. H. Sun, D. Huang, G. Liu, F. Jian, J. Zhu, and L. Zhang, "SIRT4 acts as a tumor suppressor in gastric cancer by inhibiting cell proliferation, migration, and invasion," Onco Targets Ther, vol. 11, pp. 3959-3968, 2018.

[26]. A. Csibi, S.-M. Fendt, C. Li, G. Poulogiannis, A. Y. Choo, D. J. Chapski, S. M. Jeong, J. M. Dempsey, A. Parkhitko, and T. Morrison, "The mTORC1 pathway stimulates glutamine metabolism and cell proliferation by repressing SIRT4," Cell, vol. 153, no. 4, pp. 840-854, 2013.

Cite this article as : Abdul-Nazif Mahmud, Feilun Cui, Xue Bo, Jianpeng Hu, Tingjun Liu, Muslimat Kehinde Adebis, Said Abdulrahman Salim, Aaron Gia Kanton, " SIRT4 Overexpression Promotes the Inhibition of Cell Proliferation and Invasion in Prostate Cancer", International Journal of Scientific Research in Science and Technology(IJSRST), Print ISSN : 2395-6011, Online ISSN : 2395-602X, Volume 7, Issue 3, pp.276284, May-June-2020. Available at doi : https://doi.org/10.32628/IJSRST207347 Journal URL : http://ijsrst.com/IJSRST207347 